\title{
A Fuzzy Inference Approach for the Diagnosis of Sleep Disorders
}

\author{
Vijay Kumar Garg \\ Research Scholar \\ School of Computer Applications \\ Guru Kashi University
}

\author{
R.K. Bansal \\ Professor and Dean Research \\ School of Computer Applications \\ Guru Kashi University
}

\begin{abstract}
Sleep plays a vital role in the life of human being and in convention of neuro-science. But some time, sleep is disrupted along with unusual behaviors associated with it. A numerous techniques and methods are adopted by many researchers for the diagnosis of disruptions to sleep along with the other sleep disorders and also for the diagnosis of unusual behavior linked with sleep that can also increase the sleep disruptions. In this paper, a fuzzy inference system (FIS) is developed for the diagnosis of sleep disorders like Sleep Apnea, Insomina, Parasomnia and Snoring. The dataset considered in this study is collected from various physicians. To construct the fuzzy inference system, three membership functions are used like low, medium and high. The range for all these membership functions is set according to their importance in the respective disease. A record of 140 patients is considered in this work. The accuracy achieved from the proposed system is $89.2 \%$.
\end{abstract}

\section{Keywords}

Fuzzy Logic, Membership Functions, Sleep Disorders

\section{INTRODUCTION}

Sleep is a natural state associated with the relaxation of muscles, suspension of sensational activities by generally reducing or cut off consciousness and having a narrowed attention towards environmental stimuli. A person is undergone from various stages during its sleeping hours. The architecture of sleep is distinguished by following stages: Wake Stage, Non-Rapid Eye Movement (NREM) Stage and Rapid Eye Movement (REM) Stage. The first main stage i.e. wake stage is a normal state in which a person remains always attentive and performs all daily routine tasks. NREM stage is divided into sub-stages like I) Stage 1:- The stage falls in between sleep and wake stage. It is also known as light sleep (alpha activity) due to activeness of muscles and eyes. II) Stage 2:- In this stage, theta activity produces the interruption in the alpha activity of preceding stage 1 . Though, it is tough to awake a person from this stage. III) Stage $3 \& 4$ :- The stage involved delta activity which is also termed as slow-wave sleep (SWS). A person in this stage is less susceptible towards the surroundings that's why also called as deep sleep. The most of the muscles become paralyzed at this moment. Approximately 90 minutes are taken by an adult present to reach at REM stage. The functionality of REM stage is undetermined but an inadequacy will decrease the capability to determine the complex tasks. Usually dreams occur at the time of REM sleep.

Excessive daytime sleepiness evoked by two major sleep disorders: sleep apnea [1] and narcolepsy. The sleep apnea/hypopnea syndrome is a sleep disorder, qualified by disrupted breathing during sleep that is very common among population [3]. Due to this normal sleep cycle of a person is not completed and leads to a restless sleep and it also affects the whole routine of a person. Insomnia is a sleep disorder in which a person is not enough able to fall asleep or to remain asleep as long as hoped. Parasomnia is a class of sleep disorder related to irregular and abnormal movements, behaviors, emotions, perceptions and dreams occurrence during asleep or arousal from sleep. Snoring is a sound produced due to obstructed air movement during breathing at the time of sleep. There are many kinds of other sleep disorders create effect on a person's life.

All these sleep disorders have several long duration frightful effects. The short term effects are less productivity [2], increased possibilities of accidents, loss of concentration, also disrupts daily life of a person. Long term risks are cardiovascular diseases, high blood pressure, bulkiness, type2 diabetes and memory impairment, hopelessness, increased mortality from accidents. Many of the sleep disorders are enough rigorous to slow down the physical, psychological, cognitive and motor functionality of human being. In normal life, no extra attention is paid to snoring, sleep apnea, insomnia and parasomnia but day by day all these disorders make a home in human body. So, the early diagnosis of all these disorders can reduce the above said risks. In this paper, a fuzzy inference system is proposed to classify the four sleep disorders: Sleep Apnea, Insomnia, Parasomnia and Snoring.

The rest of paper formulated as follows. Section 2 is concerned with previous studies. The materials and methodology is described in Section 3 and Section 4 deals with achieved results. Consequently, Section 5 includes the summarization of results and future work and in Section 6, all the references are mentioned.

\section{RELATED WORK}

Several techniques and methods have been enforced on physionet dataset for the diagnosis of sleep, sleep stages and sleep spindles and for many other diseases and a very high classification accuracy is achieved in these cases. Rule based reasoning plays a crucial role in drafting decisions applicable in medical era. All these rules helped us a lot to implement the fuzzy inference system (FIS). Fuzzy rule based system provides good results for some samples that still can be enhanced [4]. FIS can be implemented by two models: Mamdani-like and Sugeno Model. The Mamdani-like fuzzy model modifies the information to be represented in the form of linguistic rules that is very near to human language. So, it increases the understanding and also, allows the capability to explain the information.

In [5], authors have analyzed the macrostructure of the sleep by using fuzzy set. In this fuzzy set, 111 fuzzy rules were used along with trapezoidal fuzzy sets and for the optimization of parameters, fuzzy set is integrated with genetic algorithm. 
Estevez et. al. [6], proposed the two layer architecture for the evaluation of wake sleep state by using fuzzy set. Five parameters were undertaken in this study using trapezoidal fuzzy sets. The accuracy, sensitivity and specificity were $95.6 \%, 95.5 \%$ and 95.15 respectively. In [7], authors have presented a system for the detection of apneic events in sleep apnea/hypopnea. The sensitivity and specificity was $87 \%$ and $89 \%$ resp. In all the studies, defuzzification is executed by center of gravity method.

Pinero et. al. [8], have classified the sleep stages by using five layers architecture. GENRUL5 and MLRUL algorithms are the two algorithms used for fuzzy rules, fuzzy pack and hard rule system. In this work, CBR was used in the inference process.

In [9], authors have proposed the system for detection of apnea and hypopnea events. In this system, energy of breathing sound signals was broken down into two parts like sound and silent segments that is passed as an input to the fuzzy system. Fuzzification was done by sigmoid function. The correlation between proposed system and PSG was 96\%. The sensitivity and specificity provided by this system was above $90 \%$.

\section{MATERIAL AND METHODS}

In this study, the knowledge is acquired from various physicians. The whole data is decomposed into four factors: physical, psychological, cognitive and motor function disability. The following Table-1 represents all the symptoms considered according to the sleep disorders that affect the body part by different means. The effects of sleep disorder disease on the physical structure, psychological, cognitive and motor functions of a human being are shown in the Table- 2 . All the representation is denoted in the form of ' 0 ' and ' 1 '. Here ' 0 ' depicts that particular symptoms is not related to the prescribed disease and ' 1 ' depicts the availability of that symptoms in underlined disease.

Table-1 Symptoms of four Sleep Disorders

\begin{tabular}{|l|l|}
\hline \multirow{2}{*}{ Disorders } & \multicolumn{2}{|c|}{ Symptoms } \\
\hline Sleep & High Blood Pressure (HB), Dry Throat (DT), \\
Apnea & Bedwetting (BW), Irregular heart rhythm \\
& (IHR), Breathing (BT), Headache (HD), \\
& Unusual Sleep positions (US), Teeth \\
\hline
\end{tabular}

\begin{tabular}{|l|l|}
\hline \multirow{5}{*}{ Insomnia } & $\begin{array}{l}\text { Grinding/Clenching (TG/TC), nightmare } \\
\text { choking (NC), Napping / Drowsiness (NP), } \\
\text { Sleep Disturbances (SD), Daytime sleepiness } \\
\text { (DS), Night awakening (NA), Moodiness } \\
\text { (MD), Concentration Loss (CS), Forgetfulness } \\
\text { (FG), Learning problem (LP). }\end{array}$ \\
\hline Pry throat, Irregular heart rhythm, Eye \\
$\begin{array}{l}\text { Movement (EM), Disturbance, Daytime } \\
\text { sleepiness, Night awakening, Moodiness, } \\
\text { Tiredness (TD), Concentration Loss. }\end{array}$ \\
\hline Snoring & $\begin{array}{l}\text { Bedwetting, Irregular heart rhythm, Unusual } \\
\text { Sleep position, teeth Grinding / Clenching, } \\
\text { Eye Movement, nightmare choking, Sleep } \\
\text { Disturbance. }\end{array}$ \\
\hline $\begin{array}{l}\text { High BP, Dry throat, Irregular heart rhythm, } \\
\text { Breathing, Headache, Napping / Drowsiness, } \\
\text { Daytime sleepiness, Night awakening, } \\
\text { Learning problem. }\end{array}$ \\
\hline
\end{tabular}

\section{IMPLEMENTATION}

\subsection{Fuzzy Inference System (FIS)}

In the proposed work, Mamdani-like fuzzy inference system [11] is implemented using total four inputs that includes 81 fuzzy rules. In FIS, Trapezoidal membership function is used to specify the input variables and trimf is used to define the output variables. Three membership functions are used for each input variable like low, medium and high and four membership functions for the output variable. The trapezoidal curve depends on the four parameters expressed by the following equation:

$$
\mathrm{g}(\mathrm{n} ; \mathrm{a}, \mathrm{b}, \mathrm{c}, \mathrm{d})=\left\{\begin{array}{cc}
0, & \mathrm{n} \leq \mathrm{a} \\
\mathrm{n}-\mathrm{a} / \mathrm{b}-\mathrm{c} & \mathrm{a} \leq \mathrm{n} \leq \mathrm{b} g \\
1 & \mathrm{~b} \leq \mathrm{n} \leq \mathrm{c} \\
\mathrm{d}-\mathrm{n} / \mathrm{d}-\mathrm{c} & \mathrm{c} \leq \mathrm{n} \leq \mathrm{d} \\
0 & \mathrm{~d} \leq \mathrm{n}
\end{array}\right\}
$$

Table-2 Effects of sleep disorder diseases

\begin{tabular}{|c|c|c|c|c|c|c|c|c|c|c|c|c|c|c|c|c|c|c|c|}
\hline \multirow{2}{*}{$\begin{array}{l}\text { Sleep } \\
\text { Disorders }\end{array}$} & \multicolumn{4}{|c|}{$\begin{array}{l}\text { Motor-Function } \\
\text { Disability }\end{array}$} & \multicolumn{5}{|c|}{ Psychological Disability } & \multicolumn{3}{|c|}{$\begin{array}{l}\text { Cognitive } \\
\text { Disability }\end{array}$} & \multicolumn{7}{|c|}{ Physical Disability } \\
\hline & TG/TC & EM & $\mathrm{NC}$ & NP & SD & DS & $\mathrm{TD}$ & NA & MD & $\mathrm{CS}$ & FG & LP & HB & DT & BW & IHR & BT & HD & US \\
\hline $\begin{array}{l}\text { Sleep } \\
\text { Apnea }\end{array}$ & 1 & 0 & 1 & 1 & 1 & 1 & 0 & 1 & 1 & 1 & 1 & 1 & 1 & 1 & 1 & 1 & 1 & 1 & 1 \\
\hline Insomnia & 0 & 1 & 0 & 0 & 1 & 1 & 1 & 1 & 1 & 1 & 0 & 0 & 0 & 1 & 0 & 1 & 0 & 0 & 0 \\
\hline Parasomnia & 1 & 1 & 1 & 0 & 1 & 0 & 0 & 0 & 0 & 0 & 0 & 0 & 0 & 0 & 1 & 1 & 0 & 0 & 1 \\
\hline Snoring & 0 & 0 & 0 & 1 & 0 & 1 & 0 & 1 & 0 & 0 & 0 & 1 & 1 & 1 & 0 & 1 & 1 & 1 & 0 \\
\hline
\end{tabular}




\subsection{Structure of FIS}

The following structure of fuzzy inference system is implemented in this work in which 4 input variables are passed as an input like physical disability, cognitive disability, psychological disability and motor function disability and resultant is provided by FIS as a one sleep disorder disease according to the symptoms provided by patient.

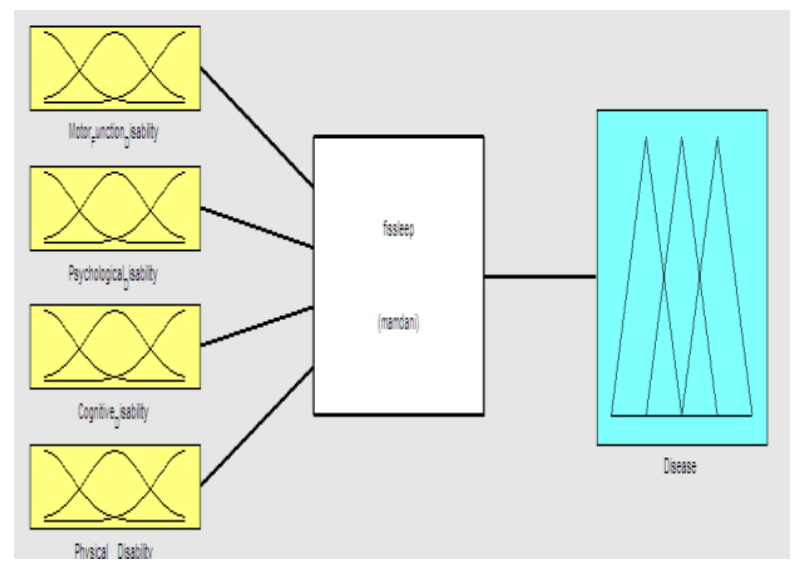

Fig.1. Structure of FIS

\subsection{Membership Functions}

The following Fig.2 represents the membership function of one of the undertaken inputs/outputs. As three membership functions (Low, medium, High) are computed in this study that all have ranges according to their importance in the respective diseases. Same membership functions are used for other input variable.

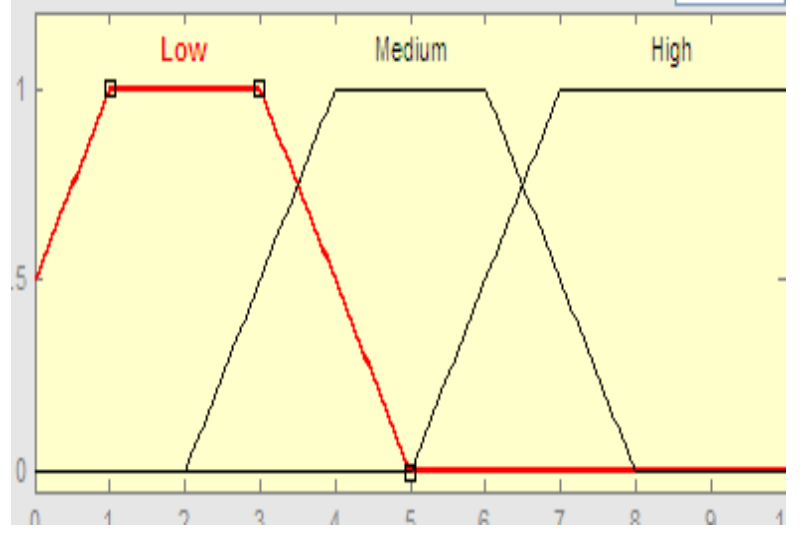

Fig. 2(a) Membership function of FIS

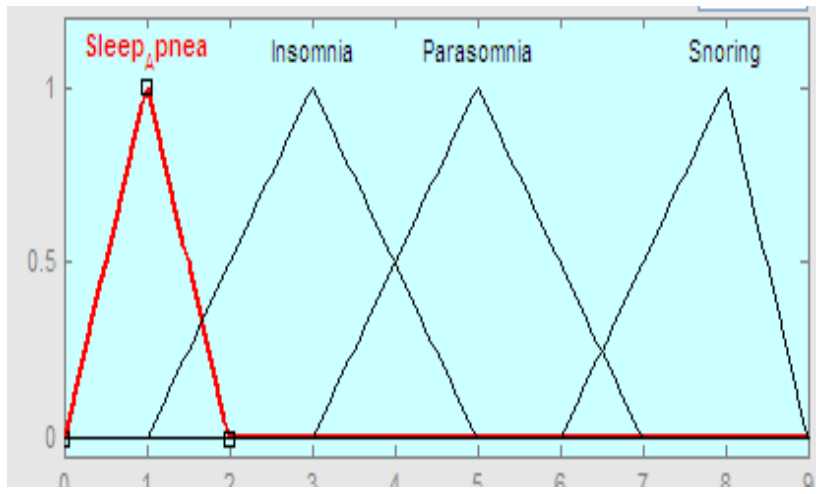

Fig. 2(b) Results of FIS

\subsection{Fuzzy Rules}

In the proposed FIS, a total of 81 fuzzy rules are implemented. A few rules are demonstrated as below. Consequently, other rules are implemented.

RULE 1: If (Motor_Function_Disability is High) and (Psychological_Disability is High) and (Cognitive_Disability is High) and (Physical__Disability is High) then (Disease is Sleep_Apnea)

RULE 2: If (Motor_Function_Disability is Low) and (Psychological_Disability is High) and (Cognitive_Disability is Medium) and (Physical__Disability is Low) then (Disease is Insomnia)

RULE 3: If (Motor_Function_Disability is High) and (Psychological_Disability is Medium) and (Cognitive_Disability is Low) and (Physical__Disability is Low) then (Disease is Parasomnia)

RULE 4: If (Motor_Function_Disability is Low) and (Psychological_Disability is Medium) and (Cognitive_Disability is Low) and (Physical_Disability is High) then (Disease is Snoring)

\section{RESULTS}

To compute the results, total 140 cases are considered, Out of which 100 cases are suffered from the sleep disorders and 40 cases have no problem of sleep disorders. The sensitivity and specificity is calculated from these 140 cases by using below formulae and it is $89 \%$ and $90 \%$ respectively. The accuracy achieved from the proposed system is $89.2 \%$.

$$
\begin{aligned}
\text { Sensitivity } & =\mathrm{TP} /(\mathrm{TP}+\mathrm{FN})(\%) \\
& =89 /(89+11)=89 \% \\
\text { Specificity } & =\mathrm{TN} /(\mathrm{FP}+\mathrm{TN})(\%) \\
& =36 /(4+36)=90 \% \\
\text { Accuracy }= & (\mathrm{TP}+\mathrm{TN}) /(\mathrm{TP}+\mathrm{TN}+\mathrm{FP}+\mathrm{FN})(\%) \\
& =(89+36) /(89+36+4+11) \\
& =89.2 \%
\end{aligned}
$$

True Positive (TP): It depicts the total number of records categorized as true while they were actually true.

False Negative (FN): It represents the total number of records found as false while they were actually true.

False Positive (FP): It represents the total number of records categorized as true while they were actually false.

True Negative (TN): It depicts the total number of records found as false while they were actually false.

\section{DISCUSSION AND FUTURE SCOPE}

A fuzzy inference system is proposed to classify the four sleep disorders: sleep apnea, insomnia, parasomnia and snoring. The dataset of 140 cases are carried out in this study to achieve the goal. FIS is constructed using three types of membership functions with the different range like low, medium and high. Trapezoidal membership function is used to pass the total 4 input variables based on some factors such as physical disability, cognitive disability, psychological disability and motor function disability. All these disabilities affect a human body by different means and ultimately, a person suffer from major disease that increase to mortality. So, to overcome all these diseases, the proposed fuzzy inference system can help a lot. Any individual can diagnose 
itself and accordingly they can take the decision to make control over them. In future, further more sleep disorders will be considered in-depth that can also helpful for an individual along with the physicians.

\section{REFERECES}

[1] G.Guimaraes, J.-H. peter, T. Penzel, A. Ultsch, A method for automated temporal knowledge acquisition applied to sleep-relaed breathing disorders, artificial Intelligence in Medicine, vol. 23, 2001, pp. 211-237.

[2] Yashar Maali, A novel partially connected cooperative parallel PSO-SVM algorithm: Study based on sleep apnea detection, in: Proceedings of IEEE Congress on Evolutionary Computation (CEC), 10-15, June 2012, pp. $1-8$.

[3] D Liu, Z Pang, SR Lloyd, A Neural Network Method for Detection of Obstructive Sleep Apnea and Narcolepsy Based on Pupil Size and EEG, IEEE Transactions on Neural Networks, vol. 19, Issue 2, 2008, pp. 308-318.

[4] Causa L., Held, C.M., Causa, J., Estevez, P.A., Perez, C.A., Chamorro, R., Garrido, M., Algarin, C., Peirano, P., Automated Sleep-Spindle Detection in Healthy Children Polysomnograms, IEEE Transactions on Biomedical Engineering, vol. 57, Issue 9, Sept. 2010, pp.2135-2146.

[5] Diego Alvarez-Estevez, Jose M. Fernández-Pastoriza , Elena Hernández-Pereira , Vicente Moret-Bonill, A method for the automatic analysis of the sleep macrostructure in continuum, Elsevier: Expert Systems with Applications, vol. 40, Issue 5, April 2012, pp. 17961803.

[6] Alvarez-Estevez D., Fernandez-Pastoriza, J.M., MoretBonillo, A continuous evaluation of the awake sleep state using fuzzy reasoning, in: Proceedings of Annual International Conference of IEEE on Engineering in Medicine and Biology Society, EMBC, 3-6 Sept. 2009, pp.5539-5542.

[7] Diego Alvarez-Estevez, Vicente Moret-Bonillo, Fuzzy reasoning used to detect apneic events in the sleep apneahypopnea syndrome, Elsevier: Expert Systems with Applications, vol. 36, Issue 4, May 2009, pp. 7778-7785.

[8] Pedro Pinero, Pavel Garciaa, Leticia Arco, Alfredo Álvarezc, M.Matilde Garciiab, Rolando Bona, Sleep stage classification using fuzzy sets and machine learning techniques, Elsevier: Neurocomputing, vol. 58-60, June 2004, pp. 1137-1143.

[9] Azadeh Yadollahi, Zahra Moussavi, Acoustic Obstructive sleep apnea detection, in: Proceedings of 31st Annual International Conference of the IEEE EMBS Minneapolis, Minnesota, USA, 2009, September 2-6.

[10] Sleep Disorder Overview. www.neurologychannel.com

[11] Sheng-Fu Liang, Ying-Huang Chen, Chih-En Kuo, JyunYu Chen, Sheng-Che Hsu, A fuzzy inference system for sleep staging, in: Proceedings of IEEE International Conference on Fuzzy Systems (FUZZ), 27-30 June 2011, pp.2104-2107. 